\title{
Prognosis of residual axillary disease after neoadjuvant chemotherapy in clinically node-positive breast cancer patients: isolated tumor cells and micrometastases carry a better prognosis than macrometastases
}

\author{
T. J. A. van Nijnatten ${ }^{1,2,3} \cdot$ J. M. Simons ${ }^{4} \cdot$ M. Moossdorff ${ }^{2,3} \cdot$ L. de Munck ${ }^{5}$ • \\ M. B. I. Lobbes' ${ }^{1}$ C. C. van der Pol ${ }^{4}$ - L. B. Koppert ${ }^{6}$ E. J. T. Luiten ${ }^{7}$. \\ M. L. Smidt $\mathbf{2}^{2,3}$
}

Received: 10 January 2017 / Accepted: 13 February 2017/Published online: 17 February 2017

(c) The Author(s) 2017. This article is published with open access at Springerlink.com

\begin{abstract}
Purpose The aim of this study was to compare disease-free survival (DFS) and overall survival (OS) between clinically node-positive breast cancer patients, treated with neoadjuvant chemotherapy (NAC), with axillary pathologic complete response (ypN0), residual axillary isolated tumor cells or micrometastases (ypNitc/mi), and residual axillary macrometastases (ypN1-3).

Methods All patients diagnosed with clinically node-positive primary invasive breast cancer treated with NAC and subsequent axillary lymph node dissection between 2005 and 2008 were retrospectively analyzed. Data were obtained from the Netherlands Cancer Registry. Patients
\end{abstract}

T. J. A. van Nijnatten, J. M. Simons authors contributed equally to this work.

$\triangle$ T. J. A. van Nijnatten

Thiemovn@gmail.com

1 Department of Radiology and Nuclear Medicine, Maastricht University Medical Center+, P.O. Box 5800, 6202 AZ Maastricht, The Netherlands

2 Department of Surgery, Maastricht University Medical Center+, Maastricht, The Netherlands

3 GROW - School for Oncology and Developmental Biology, Maastricht University Medical Center+, Maastricht, The Netherlands

4 Department of Surgery, University Medical Center Utrecht, Utrecht, The Netherlands

5 Department of Research, Netherlands Comprehensive Cancer Organisation, Utrecht, The Netherlands

6 Department of Surgical Oncology, Erasmus MC Cancer Institute, Rotterdam, The Netherlands

7 Department of Surgery, Amphia Hospital, Breda, The Netherlands were stratified by final pathological axillary status: ypN0, ypNitc/mi, or ypN1-3. The main outcome measures DFS and OS were analyzed using Kaplan-Meier survival analysis. Uni- and multivariable cox regression analyses were used to determine independent predictors for DFS and OS. Results A total of 1347 patients were included. Pathologic nodal status was ypN0 in $22.2 \%$, ypNitc/mi in $3.8 \%$, and ypN1-3 in $74.0 \%$ of patients. Overall, 5-year DFS was $57.8 \%$ and mean OS was 7.4 years. DFS and OS were comparable between ypN0 and ypNitc/mi (HR 1.38 (0.40-4.79, $p=0.613)$ and HR $0.92 \quad(0.27-3.09$, $p=0.889)$, respectively), but significantly different between ypNO and ypN1-3 (HR 1.78 (1.06-3.00, $p=0.031)$ and $\mathrm{HR} 1.70 \quad(1.07-2.71, \quad p=0.026)$, respectively).

Conclusions Clinically node-positive patients, treated with NAC, with axillary nodal status ypN0 or ypNitc/mi carry similar prognosis regarding DFS and OS. Axillary nodal status ypN1-3 is associated with a less favorable prognosis. Future studies should consider ypN0 and ypNitc/mi as one entity.

Keywords Breast cancer - Axillary lymph node . Neoadjuvant chemotherapy $\cdot$ Prognosis

\section{Introduction}

Over the past 20 years, a trend toward a less invasive approach regarding the surgical management of the axilla in breast cancer patients has been observed. Nowadays, a sentinel lymph node biopsy (SLNB) has been widely adopted for staging of early-stage clinically node-negative breast cancer [1]. In case of a sentinel lymph node (SLN) containing isolated tumor cells (ITCs) or 
micrometastases, a completion of axillary lymph node dissection (ALND) does not improve survival, nor does it reduce regional recurrence. Consequently, ALND following SLNB has been abandoned in these patients [2-4]. The ACOSOG Z0011 trial demonstrated no significant effect on prognosis when ALND is omitted in case of a SLN containing a limited number of metastases, even macrometastases, in patients treated with breast conserving therapy [3].

In clinically node-positive $(\mathrm{cN}+)$ patients, ALND is regarded as standard surgical therapy. However, increased utilization of neoadjuvant chemotherapy (NAC) results in axillary pathologic complete response (pCR) in $30-40 \%$ of patients [5]. Consequently, the value of ALND is topic of debate. Various studies demonstrated that axillary pCR after NAC is associated with improved prognosis [6-8]. Residual axillary disease has a less favorable prognosis, but it is unknown whether different degrees of residual axillary disease (i.e., ITCs, micrometastases, macrometastases) all have similar prognosis.

Hence, the purpose of this study was to compare prognosis of axillary pCR, residual ITCs, or micrometastases and residual macrometastases in $\mathrm{cN}+$ patients treated with NAC.

\section{Methods}

\section{Data collection}

In this study, all pathologically confirmed $\mathrm{cN}+$ patients diagnosed with primary invasive breast cancer and treated with NAC (with or without immunotherapy) followed by ALND between 2005 and 2008 were included. Exclusion criteria were synchronous breast cancer, primary surgical treatment, neoadjuvant radiation therapy, neoadjuvant endocrine therapy, unknown pathological nodal status, and distant metastases diagnosed within 91 days after primary breast cancer diagnosis. Patients who did not undergo ALND were also excluded.

Data were obtained from the Netherlands Cancer Registry (NCR), which is managed by the Netherlands Comprehensive Cancer Organisation (NCCO). The PALGA foundation (Pathologisch-Anatomisch Landelijk Geautomatiseerd Archief), a nationwide network and registry of histopathology and cytopathology diagnosis in the Netherlands, regularly submits reports of all diagnosed malignancies to the cancer registry. After notification, trained data collection registrars from the NCR extracted data from patients' records. Data were collected on age, tumor type, receptor status, surgical procedures, systemic therapy, adjuvant radiation therapy, and pathology results, including pathological TNM stage and tumor grade. During a 5-year period after initial diagnosis, the first of the following breast cancer events was registered: any local, regional, or contralateral recurrence or distant metastasis. Date of death or date of emigration was derived from the Municipal Personal Records Database (Basisregistratie Personen, BRP) and files until December 31, 2014 were analyzed.

Patients were stratified into three subgroups according to final pathologic axillary nodal status after completion of NAC and definitive surgery: pCR (ypN0), residual isolated tumor cells or micrometastases (ypNitc/mi), and residual macrometastases (ypN1-3).

\section{Neoadjuvant chemotherapy (NAC) with/without immunotherapy regimen}

During the study period, the Dutch national guideline of 2005 was in use [9]. This guideline recommended chemotherapy regimens consisting of five courses 5 Fluorouracil, Epirubicin, Cyclophosphamide (FEC), or six courses of Taxotere, Adriamycin, and Cyclophosphamide (TAC). In case of Her2Neu receptor (Her2) amplification, targeted therapy (trastuzumab) was recommended in addition to chemotherapy.

\section{Statistics}

Statistical analyses were performed using Statistical Package for the Social Sciences software (Version 22, IBM, Armonk, New York, USA). General characteristics between the three subgroups were compared using Chi squared test for categorical data and One-way ANOVA for continuous data, after confirmation of Levene's test for equality of variances. If Levene's test demonstrated significant differences among the population variances, Kruskall-Wallis test was used.

DFS was defined as time from diagnosis to any local (including carcinoma in situ), regional, or contralateral recurrence, distant metastasis or mortality within 5 years after the primary diagnosis. Events occurring 0-91 days after diagnosis were considered synchronous to the original tumor and were not counted as recurrence. OS was defined as the time interval between date of diagnosis and date of death, date of first event, date of last follow-up, or date of emigration.

DFS and OS for the three subgroups were calculated with Kaplan-Meier curves and compared with the log-rank test. $p$ values (two-sided) $<0.05$ were considered statistically significant. Relevant clinicopathological variables associated with DFS and OS were examined using univariable and, where applicable, multivariable Cox proportional hazards regression, with Hazard Ratio (HR) and corresponding $95 \%$ confidence intervals. 


\section{Results}

Between 2005 and 2008, 8176 patients were diagnosed with $\mathrm{cN}+$ breast cancer in the Netherlands. Patients were excluded for several reasons: 6553 patients underwent primary surgery; 204 patients did not undergo ALND; 9 patients were treated with neoadjuvant radiotherapy; 61 patients were treated with neoadjuvant endocrine therapy; and ypN status was unknown for 11 patients (Fig. 1). A final total of 1347 patients were included for this study: 299 ypN0, $51 \mathrm{ypNitc/mi}$ and $997 \mathrm{ypN1-3.}$

The incidence of $\mathrm{pCR}$ of the primary tumor was higher in patients with ypN0 compared to ypNitc/mi and ypN1-3 patients (41.1 vs. 19.6 and $7.1 \%$, respectively, $p<0.001$ ). Furthermore, lobular carcinoma was observed more often in patients with ypN1-3 than in ypNO and ypNitc/mi patients (9.4 vs. 5.4 and $3.9 \%$, respectively, $p=0.039$ ). Adjuvant radiation therapy was applied more often in ypN1-3 as compared to ypN0 and ypNitc/mi patients (92.1 vs. 80.9 and $80.4 \%$, respectively, $p<0.001$, Table 1 ).

\section{Disease-free survival}

Five-year follow-up was available for 944 patients (70.1\%; $n=206$ ypN0, $n=34$ ypNOi+/ypN1mi, $n=704$ ypN13): Recurrence occurred in 377 patients (39.9\%) and 22 patients died within 5 years $(2.3 \%)$. This resulted in a DFS event in $42.2 \%$ of the patients. DFS did not differ significantly between ypN0 and ypNitc/mi (71.8 vs. $70.6 \%$, $p=0.978)$. When DFS was compared between ypN0 and ypN1-3, a significant difference was found (71.8 vs. 53.4\%; $p=0.049$ ) (Fig. 2a).

Fig. 1 Flowchart of included patients. $c N+$ clinically nodepositive status, $S L N B$ sentinel lymph node biopsy, $A L N D$ axillary lymph node dissection, ypNO axillary pathologic complete response, ypNitc/mi axillary residual isolated tumor cells or micrometastases, $y p N 1$ 3 axillary residual macrometastases
Multivariable Cox regression analyses demonstrated no significant difference in DFS between ypN0 and ypNitc/mi (HR $1.38(0.40-4.79), p=0.613$ ), but a significant difference in DFS between ypN0 and ypN1-3 (HR 1.78 (1.06-3.00), $p=0.031$ ) (Table 2).

Furthermore, higher ypT stage (ypT 1-2: HR 2.73 (1.39-5.39), $p=0.004$ and ypT 3-4: HR 4.71 (2.35-9.43), $p<0.001)$ and higher tumor grade (HR 1.69 (1.19-2.40), $p=0.004)$ were identified as independent predictors of decreased DFS, whereas endocrine therapy was identified as independent predictor of increased DFS (HR 0.55 (0.36-0.85), $p=0.007)$.

\section{Overall survival}

Mean OS was 7.4 years (range $0.4-10$ years): 8.3 years for ypN0, 8.2 years for ypNitc/mi, and 7.0 years for ypN1-3 (Fig. 2b). OS was comparable between ypN0 and ypNitc/ mi $(p=0.875)$. However, OS was significantly lower for ypN1-3 as compared to ypN0 $(p=0.014)$.

Multivariable Cox regression analyses demonstrated no significant difference in OS between ypNO and ypNitc/mi (HR: $0.92(0.27-3.09), p=0.889)$, but a significant difference in OS between ypN0 and ypN1-3 (HR 1.70 $(1.07-2.71)), p=0.026$ ) (Table 3).

Other independent predictors of decreased OS were higher ypT stage (ypT 1-2: HR $2.40 \quad(1.32-4.36)$, $p=0.004)$ and ypT 3-4: HR $4.38(2.37-8.12), p<0.001)$ and higher tumor grade (HR $1.72(1.25-2.36), p=0.001)$. Furthermore, endocrine therapy (HR 0.49 (0.34-0.72), $p<0.001)$ was identified as an independent predictor of increased OS.

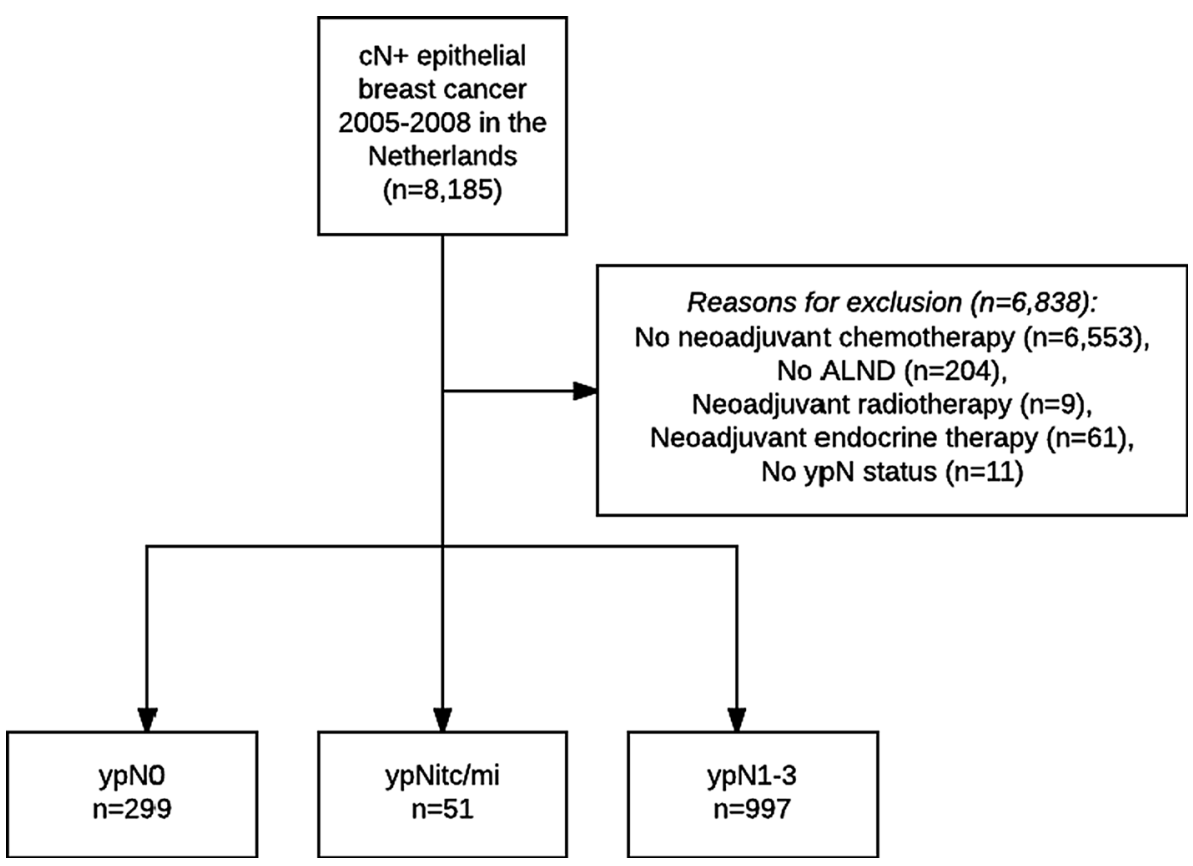


Table 1 General characteristics

\begin{tabular}{|c|c|c|c|c|}
\hline & ypN0 $(n=299)$ & ypNitc/mi $(n=51)$ & ypN1-3 $(n=997)$ & $p$ value \\
\hline Mean age (years) (range) & $48.9(27-77)$ & $48.2(29-81)$ & $50.4(22-85)$ & 0.053 \\
\hline \multicolumn{5}{|l|}{ Clinical T-stage (\%) } \\
\hline cT0-is & $1(0.3)$ & 0 & $5(0.5)$ & 0.826 \\
\hline cT1-2 & $138(47.0)$ & $26(52.0)$ & $458(47.0)$ & 0.780 \\
\hline cT3-4 & $153(52.0)$ & $24(48.0)$ & $509(52.0)$ & 0.853 \\
\hline cTx & 7 & 1 & 25 & - \\
\hline \multicolumn{5}{|l|}{ Pathologic T-stage (\%) } \\
\hline ypT0-is & $123(50.8)$ & $10(26.3)$ & $71(8.5)$ & $<0.001$ \\
\hline урТ1-2 & $107(44.2)$ & $25(65.8)$ & $570(68.5)$ & $<0.001$ \\
\hline урT3-4 & $12(5.0)$ & $3(7.9)$ & $191(23.0)$ & $<0.001$ \\
\hline Unknown & 57 & 13 & 165 & - \\
\hline \multicolumn{5}{|l|}{ Tumor grade $(\%)$} \\
\hline $1-2$ & $28(31.5)$ & $7(50.0)$ & $197(43.9)$ & $<0.001$ \\
\hline 3 & $61(68.5)$ & $7(50.0)$ & $252(56.1)$ & 0.051 \\
\hline Unknown & 210 & 37 & 548 & - \\
\hline \multicolumn{5}{|l|}{ Tumor type (\%) } \\
\hline Ductal & $227(75.9)$ & $41(80.4)$ & $746(74.8)$ & 0.739 \\
\hline Lobular & $16(5.4)$ & $2(3.9)$ & $94(9.4)$ & 0.039 \\
\hline Other $^{\mathrm{a}}$ & $56(18.7)$ & $8(15.7)$ & $157(15.8)$ & 0.470 \\
\hline \multicolumn{5}{|l|}{ Subtype (\%) } \\
\hline $\mathrm{ER}+\mathrm{PR}+$, Her2- & $35(12.8)$ & $11(22.5)$ & $349(37.3)$ & $<0.001$ \\
\hline $\mathrm{ER}+\mathrm{PR}-$, Her2- & $21(7.7)$ & $7(14.3)$ & $122(13.0)$ & 0.035 \\
\hline $\mathrm{ER}+\mathrm{Her} 2+$ & $47(17.1)$ & $20(40.8)$ & $152(16.3)$ & $<0.001$ \\
\hline ER-Her2+ & $97(35.4)$ & $5(10.2)$ & $146(15.6)$ & $<0.001$ \\
\hline Triple negative & $74(27.0)$ & $6(12.2)$ & $166(17.8)$ & 0.003 \\
\hline Unknown & 25 & 2 & 62 & - \\
\hline \multicolumn{5}{|l|}{ Breast surgery $(\%)$} \\
\hline Breast conserving therapy & $62(20.7)$ & $12(23.5)$ & $181(18.2)$ & 0.421 \\
\hline Mastectomy & $237(79.3)$ & $39(76.5)$ & $816(81.8)$ & 0.421 \\
\hline Unknown & 0 & 0 & 1 & - \\
\hline \multicolumn{5}{|l|}{ Radiation therapy (\%) } \\
\hline Yes & $242(80.9)$ & $41(80.4)$ & $918(92.1)$ & $<0.001$ \\
\hline \multicolumn{5}{|c|}{ Endocrine therapy to ER+ subtype $(\%)$} \\
\hline Yes & $95(84.8)$ & $35(92.1)$ & $600(91.5)$ & 0.080 \\
\hline \multicolumn{5}{|c|}{ Trastuzumab to Her2+ subtype $(\%)$} \\
\hline Yes & $127(92.0)$ & $19(76.0)$ & $247(89.2)$ & 0.057 \\
\hline
\end{tabular}

ypNO axillary pathologic complete response, ypNitc/mi axillary residual isolated tumor cells or micrometastases, ypN1-3 axillary residual macrometastases, cT-stage clinical tumor stage, $p T$-stage pathologic tumor stage, $E R$ estrogen, $P R$ progesterone, Her2 human epidermal growth factor receptor 2

${ }^{a}$ Including adenocarcinoma not otherwise specified, mucinous carcinoma, and mixed carcinoma

\section{Discussion}

This is the first study comparing prognosis of ypN0 with ypNitc/mi and ypN1-3 in $\mathrm{cN}+$ breast cancer patients treated with NAC. It is well known that axillary pCR is an important prognostic factor [6-8]. Residual axillary disease after completion of NAC is associated with a less favorable prognosis. However, to our knowledge, this is the first study that compares the long-term effect of different degrees of residual disease on prognosis. Our study showed that ypNO and ypNitc/mi carry similar prognosis and that ypN1-3 carries a significantly different and less favorable prognosis in terms of DFS and OS.

Current guidelines still recommend to perform ALND in $\mathrm{cN}+$ patients following NAC irrespective of axillary response $[10,11]$. However, $\mathrm{cN}+$ patients converting to 


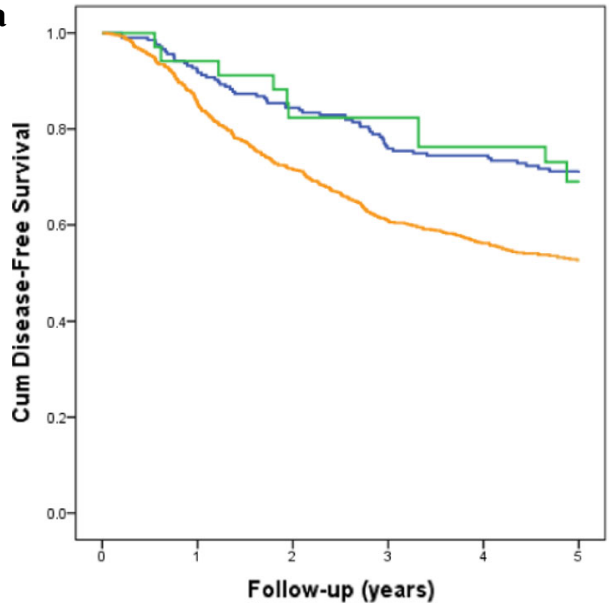

\begin{tabular}{|c|c|c|c|c|c|c|}
\hline$y p N 0$ & 206 & 188 & 172 & 152 & 146 & 80 \\
\hline$y p N i t c / m i$ & 34 & 32 & 28 & 27 & 25 & 15 \\
\hline$y p N 1-3$ & 704 & 598 & 499 & 423 & 381 & 241 \\
\hline
\end{tabular}

Fig. 2 Kaplan-Meier curves for disease-free (a) and overall survival (b), including number at risk. $y p N$ status pathologic nodal status after neoadjuvant chemotherapy, ypNO axillary pathologic complete

axillary pCR after completion of NAC remain a topic of debate since they are not expected to benefit from ALND. A non-invasive technique to accurately diagnose $\mathrm{pCR}$ is currently unavailable. Various minimally invasive procedures have been suggested for this purpose. The SLNB was studied extensively and its reliability seems questionable with a reported overall false negative rate (FNR) of $15.1 \%$ and negative predictive values (NPV) of $86 \%$ or lower [5]. Other recently introduced minimally invasive techniques, the MARI procedure (Marking the Axillary lymph node with Radioactive Iodine seeds) and TAD (Targeted Axillary Dissection), are promising with FNRs of 7 and 2\%, respectively. However, with only evidence available of single center studies comprising small cohorts that support these techniques it is not (yet) safe to implement them in clinical practice $[12,13]$.

In our cohort, all patients underwent an ALND and thus our results do not directly support a change in surgical axillary treatment after the completion of NAC. Considering the comparable prognosis between ypN0 and $\mathrm{ypNitc/mi}$, our results do question whether ypNitc/mi may mimic ypN0 more than residual axillary disease. Thus, when minimally invasive procedures prove to predict the status of the axilla accurately, the indications for omitting ALND may not just be limited to ypN0. Therefore, current research on reducing axillary management in $\mathrm{cN}+$ patients should not focus only on ypN0 patients, but also on patients with ypNitc/mi. In future, ALND may be rendered as a procedure only to manage residual macrometastases.

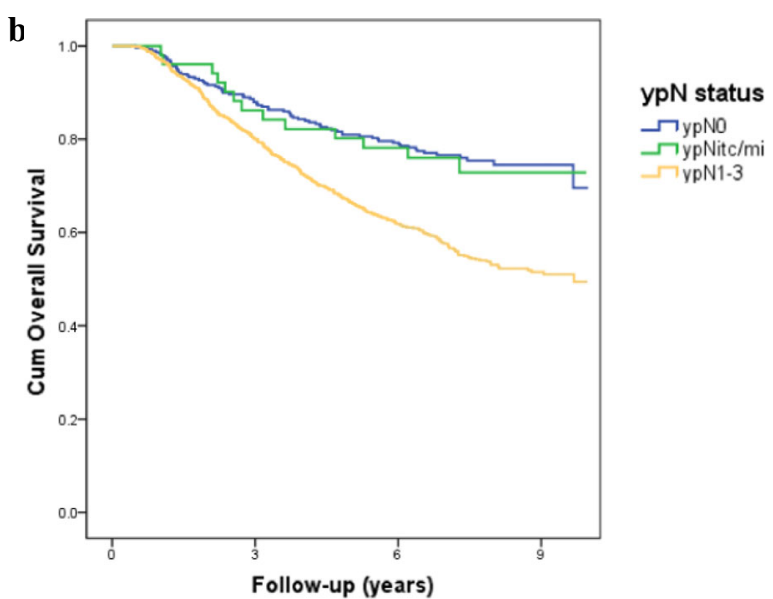

\begin{tabular}{|c|c|c|c|c|}
\hline$y p N 0$ & 299 & 263 & 236 & 43 \\
\hline$y p N i t c / m i$ & 51 & 43 & 38 & 9 \\
\hline$y p N 1-3$ & 997 & 795 & 612 & 109 \\
\hline
\end{tabular}

response, ypNitc/mi axillary residual isolated tumor cells or micrometastases, $y p N 1-3$ axillary residual macrometastases

In clinically node-negative patients in adjuvant setting, the SLNB with a relatively high FNR of about $8 \%$ is permitted since axillary recurrences are rare and previous studies have shown that not all axillary residual disease eventually converts to clinically overt disease [2, 3, 14]. This is in part effectuated by adjuvant therapy (i.e., radiation and/or systemic therapy) and by biological subtypes influencing recurrence patterns. In $\mathrm{cN}+$ patients, however, no studies have adequately evaluated prognostic impact of omitting ALND in case of residual axillary disease. Despite this, a trend toward replacing ALND by less invasive axillary staging procedures that are known to miss potentially therapy-resistant disease is already ongoing worldwide. Therefore, it is of utmost importance to prospectively collect data of these patients to detect potential influences on prognosis.

Since prognosis seems comparable between post-ALND ypNO and ypNitc/mi in $\mathrm{cN}+$ patients treated with NAC, imaging might play an important role in axillary staging after NAC in the future. Since ITCs and micrometastases are not detectable on high-resolution exams, such as MRI or 18F-FDG PET/CT, imaging techniques were considered inaccurate for nodal assessment after completion of NAC. Yet, with our current observations in mind, dedicated axillary imaging is re-entering the arena as a modality to non-invasively identify residual macrometastases rather than 'any' extent of residual disease (including ITCs and micrometastases).

The strength of the current study is the large cohort of patients that all underwent ALND after NAC. But our 
Table 2 Uni- and multivariable analyses of predictors of disease-free survival at 5 years

\begin{tabular}{|c|c|c|c|c|}
\hline & \multicolumn{2}{|c|}{ Univariable analysis } & \multicolumn{2}{|c|}{ Multivariable analysis } \\
\hline & $\operatorname{HR}(95 \% \mathrm{CI})$ & $p$ value & HR $(95 \% \mathrm{CI})$ & $p$ value \\
\hline ypN0 & Reference & 0.964 & Reference & 0.613 \\
\hline ypNitc/mi & $1.02(0.52-1.99)$ & $<0.001$ & $1.38(0.40-4.79)$ & 0.031 \\
\hline ypN1-3 & $1.89(1.43-2.50)$ & & $1.78(1.06-3.00)$ & \\
\hline Age (per year increment) & $1.02(1.01-1.02)$ & 0.001 & $1.01(0.99-1.02)$ & 0.479 \\
\hline \multicolumn{5}{|l|}{ ypT-stage } \\
\hline T0 or Tis & Reference & & Reference & \\
\hline $\mathrm{T} 1-2$ & $1.88(1.31-2.70)$ & 0.001 & $2.73(1.39-5.39)$ & 0.004 \\
\hline T3-4 & $3.74(2.53-5.54)$ & $<0.001$ & $4.71(2.35-9.43)$ & $<0.001$ \\
\hline \multicolumn{5}{|l|}{ Tumor type } \\
\hline Ductal & Reference & & Reference & \\
\hline Lobular & $0.78(0.53-1.14)$ & 0.193 & $1.11(0.59-2.07)$ & 0.751 \\
\hline Other & $0.89(0.69-1.15)$ & 0.386 & $0.89(0.58-1.37)$ & 0.595 \\
\hline \multicolumn{5}{|l|}{ Tumor grade } \\
\hline 3 versus $1-2$ & $1.64(1.22-2.20)$ & 0.001 & $1.69(1.19-2.40)$ & 0.004 \\
\hline \multicolumn{5}{|l|}{ Subtypes } \\
\hline $\mathrm{ER}+\mathrm{PR}+\mathrm{Her} 2-:$ yes versus no ${ }^{\mathrm{a}}$ & $0.63(0.50-0.80)$ & $<0.001$ & & \\
\hline $\mathrm{ER}+\mathrm{PR}-\mathrm{Her} 2-:$ yes versus no ${ }^{\mathrm{a}}$ & $1.32(0.99-1.75)$ & 0.057 & & \\
\hline ER+Her2+: yes versus no ${ }^{a, b}$ & $0.63(0.47-0.85)$ & 0.002 & & \\
\hline ER-Her2+: yes versus no ${ }^{b}$ & $1.20(0.95-1.53)$ & 0.129 & & \\
\hline Triple negative: yes versus no & $1.94(1.46-2.32)$ & $<0.001$ & $1.16(0.69-1.93)$ & 0.577 \\
\hline \multicolumn{5}{|l|}{ Trastuzumab } \\
\hline Yes versus no & $0.81(0.65-1.00)$ & 0.052 & $0.76(0.47-1.23)$ & 0.263 \\
\hline \multicolumn{5}{|l|}{ Endocrine therapy } \\
\hline Yes versus no & $0.57(0.47-0.69)$ & $<0.001$ & $0.55(0.36-0.85)$ & 0.007 \\
\hline \multicolumn{5}{|l|}{ Radiation therapy } \\
\hline Yes versus no & $1.09(0.78-1.52)$ & 0.626 & $0.74(0.45-1.23)$ & 0.251 \\
\hline
\end{tabular}

$H R$ hazard ratio, $y p N O$ axillary pathologic complete response, ypNitc/mi axillary residual isolated tumor cells or micrometastases, ypN1-3 axillary residual macrometastases, ypT-stage pathologic tumor stage after neo-adjuvant chemotherapy, $E R$ estrogen, $P R$ progesterone, Her2 human epidermal growth factor receptor 2

${ }^{a}$ Excluded from multivariable analysis due to collinearity with endocrine therapy

b Excluded from multivariable analysis due to collinearity with trastuzumab study also has several limitations. Subgroups ypN0 and ypN1-3 comprised 299 and 997 patients, respectively, where subgroup ypNitc/mi comprised only 51 patients. Our $\mathrm{ypNitc/mi}$ subcohort was too small to explore the influence of single versus multiple tumor-positive lymph nodes on prognosis, and further studies are needed to explore this concept. Yet, this subset of patients can be considered unique since $\mathrm{ypNitc/mi} \mathrm{in} \mathrm{cN}+$ breast cancer is rare and a previously reported study included only a few ypNitc/mi patients [15].

Furthermore, our cohort was treated up to a decade ago. In that time frame, different guidelines were effective, and therefore results should be interpreted carefully regarding current practice. For example, some Her2+ patients did not receive trastuzumab in our cohort $(19.6 \%)$, since trastuzumab was just introduced by that time.
Finally, our results are based on a retrospective study design. Consequently, details on additional radiation therapy could not be taken into account since radiation therapy fields were not recorded for each patient. Therefore, its influence on prognosis could not be explored in more detail.

In conclusion, our study showed that prognosis of $\mathrm{cN}+$ patients who receive NAC is affected by the degree of axillary residual disease as measured in ALND specimens. Prognosis of isolated tumor cells and micrometastases was comparable to prognosis of ypN0 and more favorable than prognosis of macrometastases in terms of DFS and OS irrespective of tumor type. Ongoing and future studies should therefore consider ypN0 and ypNitc/ $\mathrm{mi}$ as one entity. Future research must explore which patients may safely receive a different, less invasive 
Table 3 Uni- and multivariable analyses of predictors for overall survival

\begin{tabular}{|c|c|c|c|c|}
\hline & \multicolumn{2}{|c|}{ Univariable analysis } & \multicolumn{2}{|c|}{ Multivariable analysis } \\
\hline & $\mathrm{HR}(95 \% \mathrm{CI})$ & $p$ value & $\operatorname{HR}(95 \% \mathrm{CI})$ & $p$ value \\
\hline ypN0 & Reference & 0.816 & Reference & 0.889 \\
\hline ypNitc/mi & $1.07(0.59-1.94)$ & $<0.001$ & $0.92(0.27-3.09)$ & 0.026 \\
\hline ypN1-3 & $2.07(1.62-2.67)$ & & $1.70(1.07-2.71)$ & \\
\hline Age (per year increment) & $1.02(1.02-1.03)$ & $<0.001$ & $1.01(1.00-1.02)$ & 0.082 \\
\hline \multicolumn{5}{|l|}{ ypT-stage } \\
\hline T0 or Tis & Reference & 0.002 & Reference & \\
\hline T1-2 & $1.60(1.19-2.15)$ & $<0.001$ & $2.40(1.32-4.36)$ & 0.004 \\
\hline T3-4 & $3.33(2.41-4.59)$ & & $4.38(2.37-8.12)$ & $<0.001$ \\
\hline \multicolumn{5}{|l|}{ Tumor type } \\
\hline Ductal & Reference & & Reference & \\
\hline Lobular & $0.98(0.72-1.33)$ & 0.906 & $1.33(0.78-2.27)$ & 0.288 \\
\hline Other & $0.98(0.78-1.24)$ & 0.882 & $1.03(0.69-1.53)$ & 0.887 \\
\hline \multicolumn{5}{|l|}{ Tumor grade } \\
\hline 3 versus $1-2$ & $1.84(1.41-2.40)$ & $<0.001$ & $1.72(1.25-2.36)$ & 0.001 \\
\hline \multicolumn{5}{|l|}{ Subtypes } \\
\hline $\mathrm{ER}+\mathrm{PR}+\mathrm{Her} 2-:$ yes versus no ${ }^{\mathrm{a}}$ & $0.62(0.51-0.76)$ & $<0.001$ & & \\
\hline $\mathrm{ER}+\mathrm{PR}-\mathrm{Her} 2-:$ yes versus no ${ }^{\mathrm{a}}$ & $1.28(1.00-1.65)$ & 0.050 & & \\
\hline ER+Her2+: yes versus no ${ }^{a, b}$ & $0.58(0.43-0.73)$ & $<0.001$ & & \\
\hline ER-Her2+: yes versus no ${ }^{b}$ & $1.15(0.93-1.42)$ & 0.201 & & \\
\hline Triple negative: yes versus no & $2.10(1.73-2.56)$ & $<0.001$ & $1.38(0.90-2.13)$ & 0.145 \\
\hline \multicolumn{5}{|l|}{ Trastuzumab } \\
\hline Yes versus no & $0.72(0.59-0.88)$ & 0.001 & $0.70(0.46-1.07)$ & 0.101 \\
\hline \multicolumn{5}{|l|}{ Endocrine therapy } \\
\hline Yes versus no & $0.56(0.47-0.66)$ & $<0.001$ & $0.49(0.34-0.72)$ & $<0.001$ \\
\hline \multicolumn{5}{|l|}{ Radiation therapy } \\
\hline Yes versus no & $1.31(0.96-1.77)$ & 0.086 & $1.06(0.64-1.74)$ & 0.829 \\
\hline
\end{tabular}

$H R$ hazard Ratio, ypNO axillary pathologic complete response, ypNitc/mi axillary residual isolated tumor cells or micrometastases, ypN1-3 axillary residual macrometastases, y $p T$-stage pathologic tumor stage after neo-adjuvant chemotherapy, ER estrogen, $P R$ progesterone, Her2 human epidermal growth factor receptor 2

a Excluded from multivariable analysis due to collinearity with endocrine therapy

b Excluded from multivariable analysis due to collinearity with trastuzumab

approach than the current standard of performing ALND after completion of NAC in all patients that were $\mathrm{cN}+$ prior to NAC.

\section{Compliance with ethical standards}

Conflict of interest ML. Smidt received a speaker honorarium from Roche Nederland B.V. All the other authors declare that they have no conflict of interest.

Ethical standards This study complies with the current laws in the Netherlands.

Open Access This article is distributed under the terms of the Creative Commons Attribution 4.0 International License (http://crea tivecommons.org/licenses/by/4.0/), which permits unrestricted use, distribution, and reproduction in any medium, provided you give appropriate credit to the original author(s) and the source, provide a link to the Creative Commons license, and indicate if changes were made.

\section{References}

1. Pilewskie ML, Morrow M (2014) Management of the clinically node-negative axilla: what have we learned from the clinical trials? Oncology (Williston Park) 28(5):371-378

2. Galimberti V, Cole BF, Zurrida S, Viale G, Luini A, Veronesi P, Baratella P, Chifu C, Sargenti M, Intra M, Gentilini O, Mastropasqua MG, Mazzarol G, Massarut S, Garbay JR, Zgajnar J, Galatius H, Recalcati A, Littlejohn D, Bamert M, Colleoni M, Price KN, Regan MM, Goldhirsch A, Coates AS, Gelber RD, Veronesi U, International Breast Cancer Study Group Trial i (2013) Axillary dissection versus no axillary dissection in patients with sentinel-node micrometastases (IBCSG 23-01): a phase 3 randomised controlled trial. Lancet Oncol 14(4): 297-305. doi:10.1016/S1470-2045(13)70035-4 
3. Giuliano AE, Ballman K, McCall L, Beitsch P, Whitworth PW, Blumencranz P, Leitch AM, Saha S, Morrow M, Hunt KK (2016) Locoregional recurrence after sentinel lymph node dissection with or without axillary dissection in patients with sentinel lymph node metastases: long-term follow-up from the american college of surgeons oncology group (Alliance) ACOSOG Z0011 Randomized Trial. Ann Surg 264(3):413-420. doi:10.1097/SLA. 0000000000001863

4. Giuliano AE, Hunt KK, Ballman KV, Beitsch PD, Whitworth PW, Blumencranz PW, Leitch AM, Saha S, McCall LM, Morrow M (2011) Axillary dissection vs no axillary dissection in women with invasive breast cancer and sentinel node metastasis: a randomized clinical trial. JAMA 305(6):569-575. doi:10.1001/jama. 2011.90

5. van Nijnatten TJ, Schipper RJ, Lobbes MB, Nelemans PJ, BeetsTan RG, Smidt ML (2015) The diagnostic performance of sentinel lymph node biopsy in pathologically confirmed node positive breast cancer patients after neoadjuvant systemic therapy: a systematic review and meta-analysis. Eur J Surg Oncol 41(10):1278-1287. doi:10.1016/j.ejso.2015.07.020

6. Mougalian SS, Hernandez M, Lei X, Lynch S, Kuerer HM, Symmans WF, Theriault RL, Fornage BD, Hsu L, Buchholz TA, Sahin AA, Hunt KK, Yang WT, Hortobagyi GN, Valero V (2016) Ten-year outcomes of patients with breast cancer with cytologically confirmed axillary lymph node metastases and pathologic complete response after primary systemic chemotherapy. JAMA Oncol 2(4):508-516. doi:10.1001/ jamaoncol.2015.4935

7. Hennessy BT, Hortobagyi GN, Rouzier R, Kuerer H, Sneige N, Buzdar AU, Kau SW, Fornage B, Sahin A, Broglio K, Singletary SE, Valero V (2005) Outcome after pathologic complete eradication of cytologically proven breast cancer axillary node metastases following primary chemotherapy. J Clin Oncol 23(36):9304-9311. doi:10.1200/JCO.2005.02.5023

8. Meric F, Mirza NQ, Buzdar AU, Hunt KK, Ames FC, Ross MI, Pollock RE, Newman LA, Feig BW, Strom EA, Buchholz TA, McNeese MD, Hortobagyi GN, Singletary SE (2000) Prognostic implications of pathological lymph node status after preoperative chemotherapy for operable T3NOM0 breast cancer. Ann Surg Oncol 7(6):435-440
9. CBO Kwaliteitsinstituut voor de Gezondheidszorg (2005) Vereniging van Integrale Kankercentra. Guideline 'Treatment of breast cancer' (Richtlijn 'Behandeling van het Mammacarcinoom')

10. Gradishar WJ, Anderson BO, Balassanian R, Blair SL, Burstein HJ, Cyr A, Elias AD, Farrar WB, Forero A, Giordano SH, Goetz M, Goldstein LJ, Hudis CA, Isakoff SJ, Marcom PK, Mayer IA, McCormick B, Moran M, Patel SA, Pierce LJ, Reed EC, Salerno KE, Schwartzberg LS, Smith KL, Smith ML, Soliman H, Somlo G, Telli M, Ward JH, Shead DA, Kumar R (2016) Invasive breast cancer version 1.2016, NCCN clinical practice guidelines in oncology. J Natl Compr Canc Netw 14(3):324-354

11. NABON (2012) National breast cancer guideline, Oncoline. https://www.oncoline.nl/breastcancer. Accessed 23 Dec 2016

12. Caudle AS, Yang WT, Krishnamurthy S, Mittendorf EA, Black DM, Gilcrease MZ, Bedrosian I, Hobbs BP, DeSnyder SM, Hwang RF, Adrada BE, Shaitelman SF, Chavez-MacGregor M, Smith BD, Candelaria RP, Babiera GV, Dogan BE, Santiago L, Hunt KK, Kuerer HM (2016) Improved axillary evaluation following neoadjuvant therapy for patients with node-positive breast cancer using selective evaluation of clipped nodes: implementation of targeted axillary dissection. J Clin Oncol 34(10):1072-1078. doi:10.1200/JCO.2015.64.0094

13. Donker M, Straver ME, Wesseling J, Loo CE, Schot M, Drukker CA, van Tinteren H, Sonke GS, Rutgers EJ, Vrancken Peeters MJ (2015) Marking axillary lymph nodes with radioactive iodine seeds for axillary staging after neoadjuvant systemic treatment in breast cancer patients: the MARI procedure. Ann Surg 261(2):378-382. doi:10.1097/SLA.0000000000000558

14. van der Ploeg IM, Nieweg OE, van Rijk MC, Valdes Olmos RA, Kroon BB (2008) Axillary recurrence after a tumour-negative sentinel node biopsy in breast cancer patients: a systematic review and meta-analysis of the literature. Eur J Surg Oncol 34(12):1277-1284. doi:10.1016/j.ejso.2008.01.034

15. Boileau JF, Poirier B, Basik M, Holloway CM, Gaboury L, Sideris L, Meterissian S, Arnaout A, Brackstone M, McCready DR, Karp SE, Trop I, Lisbona A, Wright FC, Younan RJ, Provencher L, Patocskai E, Omeroglu A, Robidoux A (2015) Sentinel node biopsy after neoadjuvant chemotherapy in biopsyproven node-positive breast cancer: the SN FNAC study. J Clin Oncol 33(3):258-264. doi:10.1200/JCO.2014.55.7827 